\title{
Self-Assembled Binary Superlattices of CdSe and Au Nanocrystals and their Fluorescence Properties
}

Elena V. Shevchenko, ${ }^{1}$ Moritz Ringler, ${ }^{2}$ Alexander Schwemer, ${ }^{2}$ Dmitri V. Talapin, ${ }^{1 *}$ Thomas A. Klar, ${ }^{2}$ Andrey L. Rogach, ${ }^{2 \star}$ Jochen Feldmann, $^{2}$ and A. Paul Alivisatos ${ }^{1}$

The Molecular Foundry, Lawrence Berkeley National Laboratory, Berkeley, California 94720, Photonics and Optoelectronics Group, Physics Department and Center for Nanoscience (CeNS), LudwigMaximilians-Universität München, Amalienstr. 54, 80799 Munich, Germany

\section{Experimental Details of Optical Measurements:}

Time-integrated fluorescence spectra were recorded at room temperature with a Cary Eclipse spectrofluorimeter (Varian). Time-resolved fluorescence spectroscopy was performed on a Picoquant Fluotime 200 spectrometer. The samples were excited with pulsed laser light $\left(\lambda_{e x}=405 \mathrm{~nm}\right)$ from a Picoquant LDH-P-C-405 pulsed diode laser at an angle of incidence of $60^{\circ}$. Repetition rates of $2 \mathrm{MHz}$ (cw power of $7.8 \mu \mathrm{W}$ ) and $10 \mathrm{MHz}$ (cw power of $18 \mu \mathrm{W}$ ) were used. The fluorescence was collected at $90^{\circ}$. Scattered excitation light was blocked with a Schott GG 435 glass filter. The fluorescence light was spectrally resolved with a Science-Tech 9030 monochromator set to $\lambda_{\mathrm{em}}=670 \mathrm{~nm}$ (slit widths: $8 \mathrm{~nm}$ ) and detected with a fast photomultiplier tube. Fluorescence photons were counted with a Picoquant Timeharp 200 TCSPC board for 300 seconds. The obtained fluorescence decay curves were corrected for dark counts and the impulse response and normalized to their peak values.

We report optical measurements for $\mathrm{AlB}_{2}$-type $\mathrm{CdSe}$ - $\mathrm{Au}$ binary nanoparticle superlattices because this structure can grow on $\mathrm{Si} / \mathrm{SiO}_{2}$ substrates in form of phase-pure crystals with micron large long-range ordered domains. Such sample quality is sufficient for unambiguous structural assignment and accurate optical measurements. Other types of $\mathrm{CdSe}-\mathrm{Au}$ BNSLs with $\mathrm{CuAu}, \mathrm{CaCu}_{5}$ and cub- $\mathrm{AB}_{13}$ structures typically form sub-micron domains. These structures often coexist making accurate far field optical measurements difficult. We strongly believe that further progress in growing BNSLs and development of BNSL characterization techniques will help establishing detailed structure-properties relationships for BNSLs self-assembled from different semiconducting and metallic nanocrystals. 


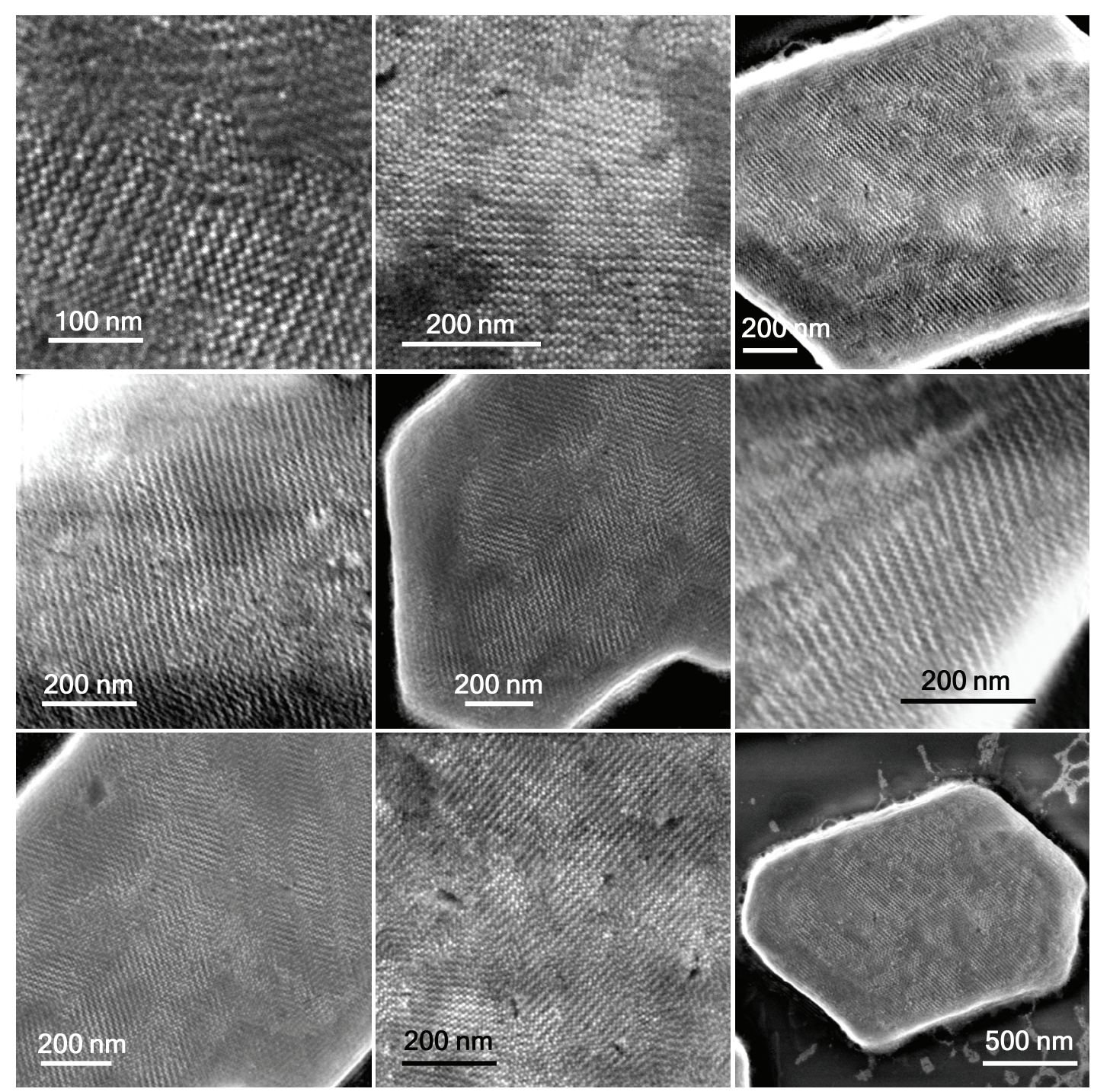

Figure S1. SEM images of AlB ${ }_{2}$-type superlattices self-assembled from $8.7 \mathrm{~nm}$ CdSe and 5.5 $n m A u$ NPs. 


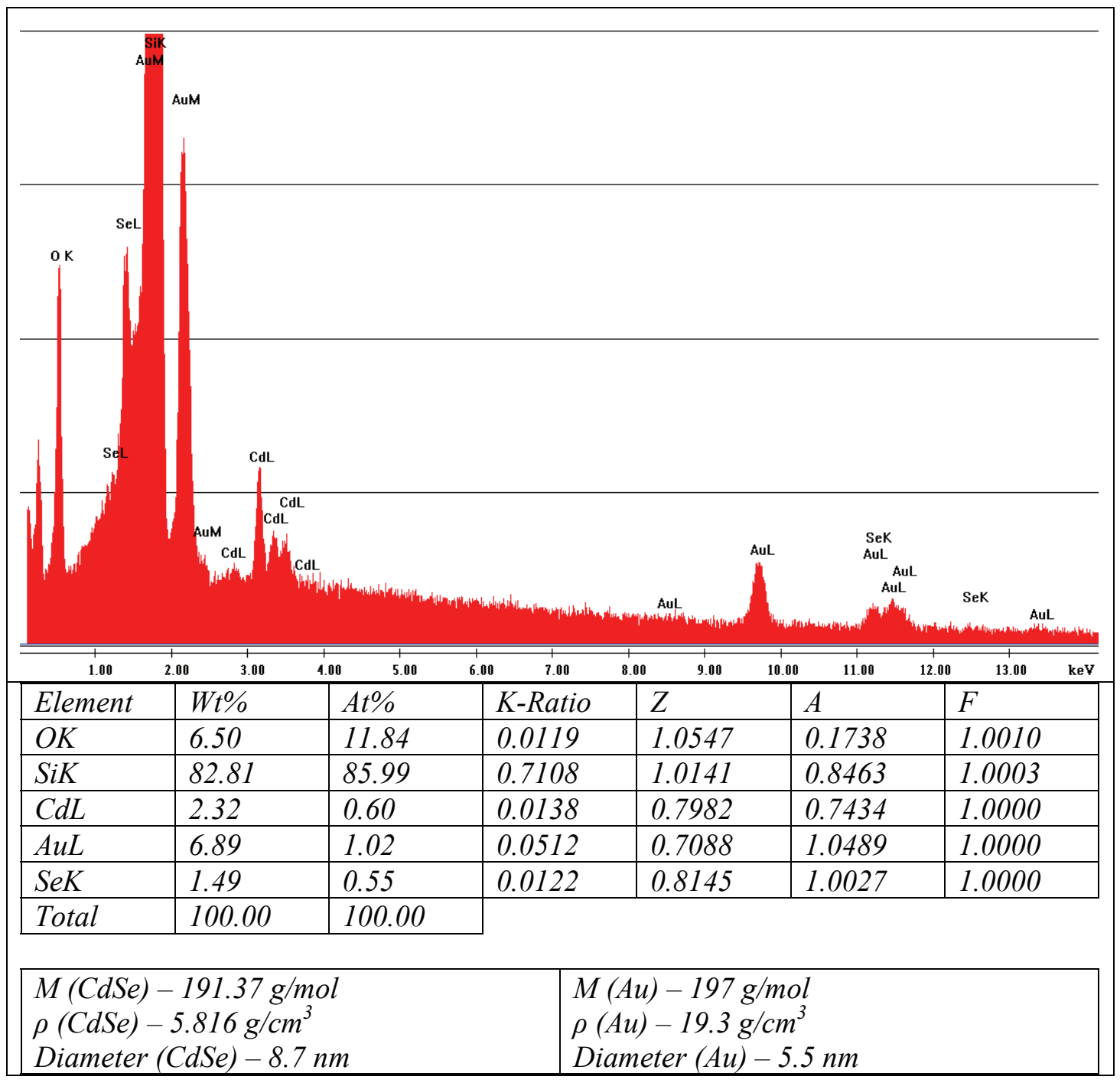

Figure S2. Energy dispersive X-ray (EDAX) analysis acquired from BNSL domain confirming its $\mathrm{AB}$ stoichiometry. 